\title{
On the optimal design of sliding rotary vane pump for heavy-duty engine cooling systems
}

\author{
Fabio Fatigati ${ }^{*}$, Davide Di Battista, Marco Di Bartolomeo, Luigi Mariani, and Roberto \\ Cipollone \\ ${ }^{1}$ University of L'Aquila, Department of Industrial and Information Engineering and Economics, \\ 67100, L'Aquila, Italy
}

\begin{abstract}
Presently the on-the-road transportation sector is responsible of the $21 \%$ of the whole $\mathrm{CO}_{2}$ amount emitted into atmosphere. This pushes the International Governments and Organizations to provide strict limitations in terms of ICEs emissions, also introducing fees payment for the car manufacturers. The vehicle electrification allows certainly to meet these requirements, but the higher cost and the need of a green electricity still limit a widespread diffusion among all social classes. Thus, the technological improvement of internal combustion engine plays a key role in the transition period. Among these technologies, the engine thermal management allows to achieve a good compromise between the $\mathrm{CO}_{2}$ emission reduction and related costs. It was demonstrated that replacing the conventional centrifugal pump of engine cooling system with a sliding vane rotary pump (SVRP), important benefits in terms of $\mathrm{CO}_{2}$ emission reduction can be achieved as centrifugal pump efficiency decreases significantly when the engine works far from the maximum load (i.e. design point of the pump). Nevertheless, the complex thermo-fluid-dynamic phenomena taking place inside a SVRP make its design not immediate, particularly if heavy duty ICE cooling systems are considered. These applications indeed are challenging due to the wide operating range and the huge flow rates which pump must deliver. These operating requirements make difficult the choice of the main design parameters: among the different ones, the pump revolution speed and displaced volume. In the present paper a design strategy is developed for this type of pumps based on a comprehensive mathematical model of the processes occurring, predicting volumetric, indicated and mechanical efficiencies. The model was validated with a wide experimental activity so acting as virtual development platform. The results show how the best global efficiency (0.59) is achieved adopting a dual axial intake port configuration, with a suitable choice result of a trade-off between displaced volume and revolution speed. The analysis also show that the pump keeps its efficiency close to the design one for a wide operating range which is particularly suitable for the cooling of an ICE.
\end{abstract}

\footnotetext{
* Corresponding author: fabio.fatigati@univaq.it
} 


\section{Introduction}

Technological advancements in the on-the-road transportation sector are needed to meet the targets imposed on harmful species and $\mathrm{CO}_{2}$. Heavy duty diesel engines in particular, account for $6 \%$ of the total $\mathrm{CO}_{2}$ emissions in Europe [1]. From 2025 onwards, a 15\% emission reduction compared to the average level achieved in the period 2019-2020 has been targeted [2]. This issue determines the necessity to improve the efficiency of these systems.

Among the different possibilities, the optimization of the engine cooling system could allow a potential reduction of $\mathrm{CO}_{2}$ emissions improving the fuel economy. Integrating inside the concept of optimization also that of the engine thermal management, a net benefit is obtained also concerning the harmful emissions.

The most important component of the cooling circuit is the cooling pump which is also responsible for some of the engine parasitic losses. Traditionally, it is mechanically coupled to the engine shaft with a certain transmission ratio which constrains the pump speed. Centrifugal pumps represent the vast majority in the automotive sector for engine cooling applications. They offer good performances and long-time known design methodologies. Most relevant studies focused the attention on cavitation phenomena through experimental and CFD analysis [3]- [5].

The electric actuation of this component has the possibility to improve the system overall performances as well as the engine fuel economy. Major advantages are represented by the possibility to decouple the pump from the engine shaft allowing a more intelligent thermal management based on the engine thermal needs. In [6] it is reported that approximately $3 \%$ lower consumption can be achieved in a compression ignition engine. The electric actuation could allow also a more flexible packaging in relation to the engine compartments and a lower radiator area due to the optimized thermal performances of the sub-system [7]. Moreover, the possibility to control the pump even after the vehicle shut-down can prevent after-boiling phenomena [8] and avoid radiator fan switch-on.

Volumetric pumps show a different behavior compared to their dynamic counterparts. Their main advantage is represented by the capability to provide flowrate almost independently from the pressure difference between inlet and outlet ports, showing a constant efficiency at different speed. The main drawback is represented by volumetric and mechanical losses, caused by the contact between moving parts. For these reasons, their use has been limited to the engine lubricating circuits, where the oil can prevent the wear of the moving parts. In these applications, variable displacement vane pumps have been frequently studied to avoid the use of a relief valve when the engine operating conditions determine an excessive flowrate of oil. This kind of systems allows a reduction of the flowrate which determines an increase of the fuel economy [9]. Several studies focused the attention on the actuator characteristics [10], [11] and on the forces acting on the components of the pump [12], [13]. In [14] and [15] the driving and friction torque of a vane pump has been experimentally and numerically analyzed, finding that the design optimization of the ratio between the eccentricity of the machine and the blade thickness ensures to increase the mechanical efficiency without affecting the volumetric performances. Different approaches have been used to model this type of pumps. Harrison et al. [16] developed a CFD-1D model showing good performances in terms of accuracy and computational cost. A similar model has been used also by the authors for the evaluation of the best control strategies [17] to control the flowrate. A different approach has been used in [18], where a deterministic model has been built which was able to reproduce the flowrate of a variable displacement vane pump with an accuracy between $1 \%$ and $7 \%$ depending on its rotational speed. 
As previously mentioned, few studies investigated the potential of variable displacement pumps for engine cooling applications. In [19] the authors designed, modelled and built a sliding vane pump for a specific engine, showing that a lower mechanical energy is absorbed by the volumetric pump which ultimately translate in a saving of $0.5 \mathrm{gCO}_{2} / \mathrm{km}$ over a typeapproval cycle for a light duty and a passenger car. The benefits are evidenced also in [20] where a $12 \%$ mechanical energy reduction is achieved which increases up to $22 \%$ if the eccentricity control is actuated. A further step in the design optimization of these components is outlined in [21] where a CFD 1D numerical model has been used as a design software platform to understand the influence of the inlet and outlet ports shape and configurations. Results show that an economy of $1.4 \mathrm{gCO}_{2} / \mathrm{km}$ can be achieved.

In the present paper, a further contribution is given to the optimization of design of SVRP employed for the engine cooling. A heavy-duty ICE is considered as case studied because this application is retained very challenging for volumetric pump. Indeed, the high flow rate to be sent and the wide operating range to be covered can involve a pump performance reduction, if the design is not correctly addressed in terms of selection of displaced volume and revolution speed of the pump. In this specific application (heavy duty), often a pump design must fulfill different engines with highly different cooling needs in terms of flow rate delivered.

The choice of high revolution speeds allows, certainly, to downsize the machine with a saving on space required on board, but severe performance penalties can be observed in this case. Friction losses, for instance, significantly increase with revolution speed, reducing overall pump efficiency and, mainly, operating reliability. Moreover, indicated efficiency decreases as well, due to the pressure losses at the suction and during the discharge. The simplest solution to reduce operative speed is to increase the displaced volume but the machine dimension could be unsuitable considering the vehicle space availability and shaping of the pump. Costs increase as well when compared to centrifugal pumps which are cheap and intrinsically reliable but with an efficiency strongly dependent on revolution speed. This causes that, even though they are designed at BEP (Best Efficiency Point) with high efficiency, their real operating regimes are far from this optimum design, wasting energy.

Therefore, with the aim to develop optimal design criteria for SVRP, after a preliminary theoretical design, a comprehensive model developed and experimentally validated by the authors in [21] is used as design tool to optimize pump design. Intake and discharge ports have been designed to optimize the indicated efficiency, being the choice of considering two intake and discharge ports opened the way to a sensible efficiency increase. Furthermore, a proper choice of pump displaced volume and revolution speed allows to simultaneously reduce the mechanical and volumetric losses.

\section{Materials and methods}

\subsection{Case study and pump preliminary design}

The FPT Industrial Cursor 13-NG has been selected as the target engine for the case study. It can run with CNG (Compressed Natural Gas), or LNG (Liquefied Natural Gas), with a stoichiometric combustion.

In Table 1 the main technical specifications of the engine have been reported. It delivers a power of up to $338 \mathrm{~kW}$ at $1900 \mathrm{rpm}$ and torque up to $2000 \mathrm{Nm}$ at $1100 \mathrm{rpm}$. Knowing the hydraulic impedance of the engine (Figure 1), the design point is selected in correspondence of maximum engine load when the engine reverses on the coolant the maximum thermal power. Therefore, in this condition the pump must provide a coolant flow rate equal to 460 
1/min whit a pressure rise of $190 \mathrm{kPa}$.

Table 1. FPT Industrial Cursor 13-NG specifications.

FPT Industrial Cursor 13-NG

\begin{tabular}{rc} 
Certification: & EURO VI E \\
No. of cylinders - valves: & $6.0-4$ \\
Injection system: & MPI (Multi-Points injection) \\
Aspiration: & WG (Fixed geometry turbocharger with Waste Gate valve) \\
Displacement: & 12.91 \\
Bore per stroke: & $135 \times 150 \mathrm{~mm}$ \\
Maximum power: & $338 \mathrm{~kW} @ 1900 \mathrm{rpm}$ \\
Maximum torque: & $2000 \mathrm{Nm} @ 1100 \mathrm{rpm}$ \\
Dry weight: & $1150 \mathrm{~kg}$ \\
Dimensions $[\mathrm{L} \times \mathrm{W} \times \mathrm{H}]:$ & $1610 \times 1027 \times 1178 \mathrm{~mm}$ \\
ATS (After Treatment System): & TWC $($ Three-Way Catalyst $)$ \\
\hline
\end{tabular}

Figure 1. Engine hydraulic impedance and design point

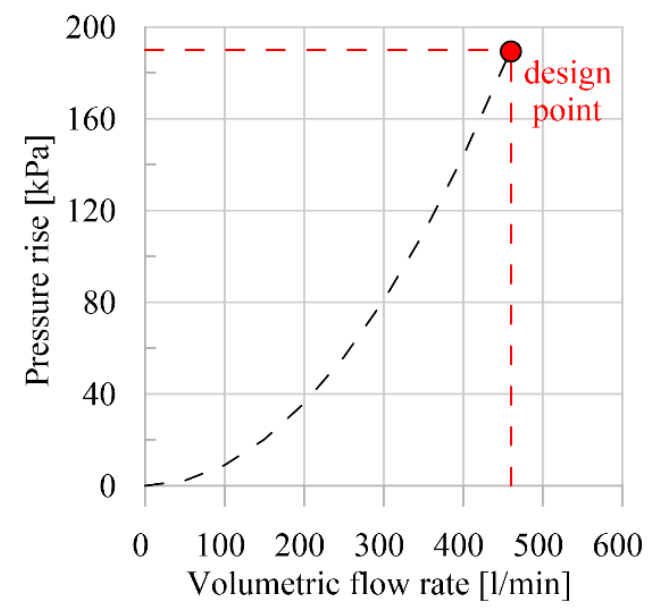

Once the design point and, consequently, the flow rate to be delivered is known, the definition of the displaced volume (the volume swept in a complete pump rotation) can be defined through Eq. (1):

$$
V_{\text {disp }}=\frac{60 Q}{\omega \eta_{v o l}}
$$


Where $Q$ is the volumetric flow rate $(1 / \mathrm{min})$ and $\omega$ is the pump revolution speed measured in rpm.

When Eq. (1) is used to to evaluate $V_{\text {disp }}$, a reasonable value for the volumetric efficiency $\eta_{\text {vol }}$ should be assumed and a pump revolution speed chosen. It is worth to observe that Eq. (1) is not linear as $\eta_{\mathrm{vol}}$ depends on $\omega$ [22], but for a preliminary design they can be retained independent each other. Indeed, a design verification was provided by a detailed numerical model developed by the authors in [21] which fully describes the processes inside the pump. Nevertheless, the choice made in this preliminary phase in terms of $\omega$ is fundamental as the will guide the design of the machine. High revolution speed allows to reduce $V_{\text {disp }}$ and then the pump dimension and weight, but, at the same time, it leads to higher friction losses. As matter of fact, these are due by the centrifugal force which pushes the blades against the stator inner surface. Friction force depends on $\omega^{2}$ (Eq. 2) which can be consequently retained the main driver of these losses:

$$
P_{\text {losses }}=f N_{v}\left(F_{c}+F_{p}\right) r_{v} \omega=f N_{v}\left(m_{b} \omega^{3} r_{v}^{2}\right)+f N_{v}\left(\left(p_{u b}-p_{a m b}\right) A_{u b}\right) \omega r_{v}
$$

Where:

- $\quad N_{v}$ is the blade number;

- $\quad r_{v}$ is the actual distance between blade tip and rotor center $[\mathrm{m}]$;

- $\quad F_{c}$ is the centrifugal force $[\mathrm{N}]$;

- $\quad F_{p}$ is the force exerted by the fluid under the blades [N];

- $\quad m_{b}$ is the blade mass $[\mathrm{kg}]$;

- $f$ is the friction factor;

- $\quad p_{\mathrm{ub}}$ is the pressure under the blades $[\mathrm{Pa}]$;

- $\quad p_{\mathrm{amb}}$ is the pressure under the blades $[\mathrm{Pa}]$;

- $\quad A_{\mathrm{ub}}$ is the area under the blades $\left[\mathrm{m}^{2}\right]$;

On the $\mathrm{P}_{\text {losses }}$ definition concurs also the force produced by the pressure exerted by the fluid under the blades inside the rotor slot. 
On the other hand, a reduction of the friction losses invites to decrease the design revolution speed but the machine dimension grows up till to assume unsuitable value in terms of space available on board.

Therefore, to compare the different design configurations two revolution speeds are considered: $2250 \mathrm{rpm}$ and $1800 \mathrm{rpm}$.

Displaced volumes equal to 0.2191 and 0.2731 are obtained respectively adopting a revolution speed equal to $2250 \mathrm{rpm}$ (HS-High Speed Pump) and $1800 \mathrm{rpm}$ (LS-Low Speed Pump). For the sake of confidentially, the pump geometry which allows to reach these displaced volumes are not directly reported but the dimensionless geometries are shown in table 2:

Table 2. Sliding Rotary Vane Pump configurations.

\begin{tabular}{|r|c|c|}
\hline & High Speed Pump & Low Speed Pump \\
\hline Design speed $\boldsymbol{\omega}$ & $\mathbf{2 2 5 0} \mathbf{~ r p m}$ & $\mathbf{1 8 0 0} \mathbf{~ r p m}$ \\
\hline Displaced volume & 0.2191 & 0.2731 \\
\hline $\mathrm{D}_{\mathrm{st}} / \mathrm{D}_{\mathrm{rt}}$ (stator diameter/stator rotor) & 1.18 & 1.21 \\
\hline $\mathrm{D}_{\mathrm{st}} / \mathrm{W}_{\mathrm{p}}$ (stator diameter/pump width) & 1.89 & 1.94 \\
\hline $\mathrm{D}_{\mathrm{st}} / \mathrm{b}_{\mathrm{l}}$ (stator diameter/blade length) & 4.16 & 4.04 \\
\hline $\mathrm{b}_{\mathrm{l}} / \mathrm{b}_{\mathrm{t}}$ (stator diameter/pump width) & 6.13 & 6.50 \\
\hline
\end{tabular}

Table 2 shows as the dimensionless ratio corresponding to the LS pump is slightly higher than the HS pump. Indeed, a higher displaced volume is achieved with an increase of $2.9 \%$ of the stator diameter. This is due to the high impact on volume increase $(+25 \%)$ provided by the increase of the stator and rotor diameters $(+3 \%)$. This is a fundamental feature of SVRP as allows to reach higher displaced volume with a slight increase of pump dimension. This makes possible the reduction of revolution speed without adopting unsuitable geometry in terms of space required on board and weight.

\subsection{Mathematical model of Sliding Rotary Vane Pump}

The two pump configurations (HS and LS) obtained from the preliminary design were verified in terms of performances by means of a SVRP comprehensive model. The model was developed and experimentally validated by the authors in [21]. It was built in GT-Suite ${ }^{\mathrm{TM}}$ environment following an integrated approach between a mono (1D) and zero (0D)dimensional thermo-fluid-dynamic analysis. The (1D) analysis was applied to represent the transient phenomena which takes place in correspondence of intake and exhaust pipes. They were discretized in multiple sub-volume and for each sub-volume the mass, momentum and energy conservation equations are solved. The (0D) approach was instead adopted to reproduce chambers filling. Each chamber is treated indeed as a lumped capacity and the volume variation is impressed by the angular position of the pump shaft which gives to the system a periodic nature. The $0 \mathrm{D}$ approach allows also to determine the leakages flows 
related to the clearance gap between blade tip and stator inner surface, blade side and rotor slot and rotor face and machine casing, [21].

The evaluation of leakages flows allows to define the volumetric efficiency of the machine as the ratio of actual and theoretical flow rate (3).

$$
\eta_{v o l}=\frac{Q}{Q_{t h}}=\frac{60 Q}{\omega V_{\text {disp }}}
$$

Leakages highly affect the indicated diagram, representing the pressure inside the chamber as function of the rotation angle. The SVRP model, in fact, can reproduce how the pressure varies during the different phases (intake and exhaust) thus assessing the power exchanged between the machine and the fluid. The corresponding indicated power $P_{\text {ind }}$ can be calculated as the area of $p$ - $V$ cycle. The $p$ - $V$ cycle (called also indicated cycle) represents the pressure $p$ trend as function of Volume $V$ (observed in a cycle) for each $i$-chamber of the pump (Eq. (4)):

$$
P_{\text {ind }}=\frac{\sum_{i=1}^{N_{v}} \oint p_{i} d V_{i}}{t_{\text {cycle }}}
$$

The hydraulic power can be defined as in (5):

$$
P_{h y d}=Q \Delta p
$$

Eqs. (3) and (5) show that leakages besides volumetric losses also involve a power penalty. Indeed, the leakages flow $Q_{\text {leak }}$ is compressed by the pump but not send. Thus, considering also the presence of leakages in (5) the following relation could be obtained:

$$
P_{h y d}^{\prime}=\left(Q+Q_{\text {leak }}\right) \Delta p=Q_{t h} \Delta p
$$

$P^{\prime}$ hyd allows to represent the increase of hydraulic power due to the flow leakages $Q_{\text {leak }}$. It is interesting to observe how the ratio between $P_{h y d}$ and $P^{\prime}{ }_{h y d}$ is equal to the volumetric efficiency Eq. (7) demonstrating the dual aspect of volumetric losses as indicated in Eq. (7).

$$
\frac{P_{h y d}}{P_{h y d}^{\prime}}=\frac{Q \Delta p}{Q_{t h} \Delta p}=\eta_{v o l}
$$

In ideal conditions the pressure inside the vane remains constant during emptying and discharging equal to the inlet and outlet pressure of the pump; pressurization occurs instantaneously following an isochoric transformation.

As it is known, many real processes deviate from this ideal behaviour. Pressure during vane filling and emptying doesn't remain constant and when the fluid crosses the ports, a pressure decrease happens as well as a pressure increase during discharging and pressure peaks are also present considering the vane geometry which doesn't match fluid incompressibility. Partial vaporization of the fluid can also happen during intake phase which can suffer of severe pressure losses with consequent re-compression of vapor pockets and waste of energy, [21]. These losses can be considered through the indicated efficiency defined as in equation (8):

$$
\eta_{\text {ind }}=\frac{P_{\text {hyd }}^{\prime}}{P_{\text {ind }}}
$$


By adding the friction losses Eq. (2) to the indicated power the whole mechanical power can be evaluated as follows:

$$
P_{\text {mech }}=P_{\text {ind }}+P_{\text {losses }}
$$

A mechanical efficiency can be also defined to highlight the impact of friction losses on the mechanical power.

$$
\eta_{\text {mech }}=\frac{P_{\text {ind }}}{P_{\text {mech }}}
$$

Multiplying volumetric, mechanical and indicated efficiency, the global performance of the machine can be assessed:

$$
\eta_{\text {glob }}=\frac{P_{\text {hyd }}}{P_{\text {hyd }}^{\prime}} \frac{P_{\text {hyd }}^{\prime}}{P_{\text {ind }}} \frac{P_{\text {ind }}}{P_{\text {mech }}}=\eta_{\text {vol }} \eta_{\text {ind }} \eta_{\text {mech }}
$$

The definition of global efficiency (10) allows to represent the efficiency chain and define the impact of mechanical or fluid-dynamic losses on pump performance.

\section{Results}

The preliminary design provided two possible pump geometries (HS and LS), according to the choice on the revolution speed. For each geometry, both radial and dual axial port configuration are considered. The radial configuration (Figure 2(a)) involves the introduction of an intake and exhaust grooves on the casing to ensure that the filling and emptying phase takes place radially. The dual axial port configuration (Figure 2(b)), on the other hand, is adopted since in [21] it was assessed that mono axial intake/exhaust port is a limiting aspect for the pump performance. Indeed, a single axial port is in general too small thus behaves like as nozzle providing an acceleration of working fluid entering the pump with a consequent a pressure reduction. It can lead to a partial vaporization of working fluid which causes an increase of indicated power due to the recompression of vapor pockets. An additional loss is also introduced considering that the vane filling happens at a pressure lower than the one at pump intake. In [21] it is also shown as also for exhaust phase an axial port is not a suitable choice. In fact, its area is not sufficient to guarantee a correct and fast exhaust phase to avoid overpressure which penalizes the indicated power.

Figure 2. Radial port (a) and Dual axial port (b) configurations of SVRP 


\section{(a)- Radial Port}

Radial

Intake
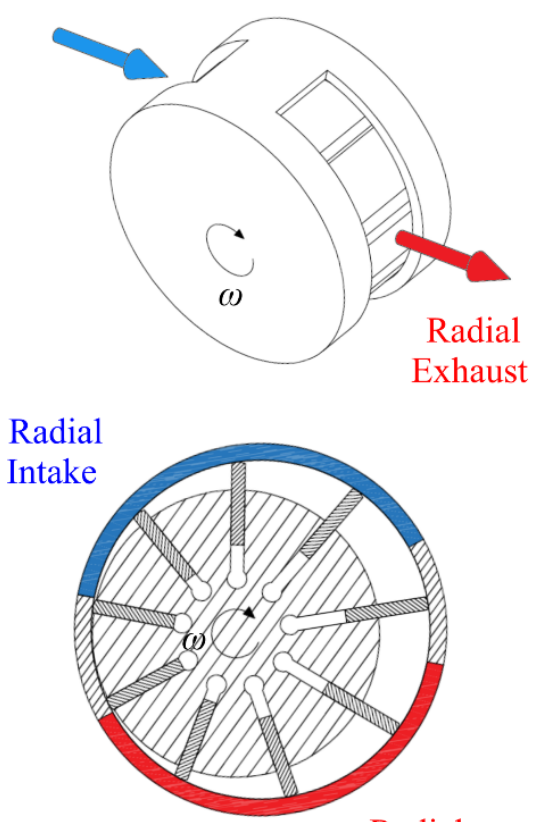

Radial (b)- Dual Axial Port

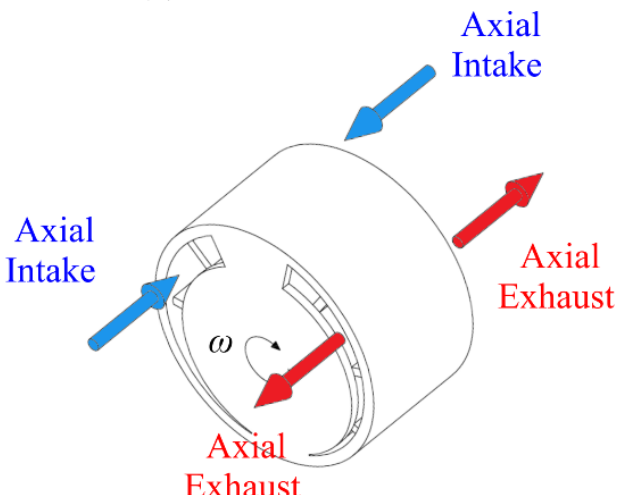

Axial

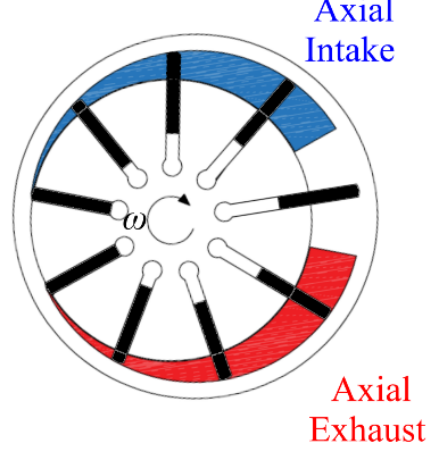

For these reasons, a dual axial port is proposed, thus the intake and exhaust phase take place on both rotor faces (Figure 2(b)). So, two streams fill the vane axially through the ports diametrically opposed; similarly the voiding of the vane through the discharge ports. In this way an effective filling and emptying happen without significant pressure losses. This makes possible an increase of the intake and exhaust phase without the introduction of groove on the stator.

Therefore, the following 4 cases have been considered for the present analysis:

(A) High Speed pump with Radial Port (HSRP);

(B) High Speed pump with Dual Axial Ports (HSDAP);

(C) Low Speed pump with Radial Port (LSRP);

(D) Low Speed pump with Dual Axial Ports (HSDAP);

Table 3 reports the machine properties and their performance, as predicted by the model.

Table 3. Performance comparison of the SVRPs configuration.

\begin{tabular}{|c|c|c|c|c|}
\hline & HSRP & HSDAP & LSRP & LSDAP \\
\hline Case & $\mathrm{A}$ & $\mathrm{B}$ & $\mathrm{C}$ & $\mathrm{D}$ \\
\hline $\begin{array}{c}\text { Displaced } \\
\text { volume [1] }\end{array}$ & 0.219 & 0.219 & 0.273 & 0.273 \\
\hline
\end{tabular}




\begin{tabular}{|c|c|c|c|c|}
\hline $\begin{array}{c}\text { Revolution } \\
\text { speed [rpm] }\end{array}$ & 2250.00 & 2250.00 & 1800.00 & 1800.00 \\
\hline $\begin{array}{c}\text { Port } \\
\text { configuration }\end{array}$ & Radial port & Dual Axial Port & Radial Port & Dual Axial Port \\
\hline $\begin{array}{c}\text { Intake } \\
\text { pressure } \\
{[\mathrm{kPa}]}\end{array}$ & 160 & 160 & 160 & 160 \\
\hline $\begin{array}{c}\text { Exhaust } \\
\text { pressure } \\
{[\mathrm{kPa}]}\end{array}$ & 350 & 350 & 350 & 350 \\
\hline $\begin{array}{c}\text { Flow rate } \\
\text { [L/min] }\end{array}$ & 478.12 & 469.15 & 476.36 & 468.92 \\
\hline $\begin{array}{c}\text { Hydraulic } \\
\text { Power [W] }\end{array}$ & 1514.05 & 1485.63 & 1508.47 & 1484.91 \\
\hline $\begin{array}{c}\text { Indicated } \\
\text { Power [W] }\end{array}$ & 1934.3 & 1986.48 & 1971.91 & 1848.5 \\
\hline $\begin{array}{c}\text { Friction } \\
\text { Power [W] }\end{array}$ & 1031.35 & 975.13 & 773.15 & 719.92 \\
\hline $\begin{array}{c}\text { Mechanical } \\
\text { Power [W] }\end{array}$ & 2965.65 & 2961.62 & 2745.06 & 2568.42 \\
\hline $\begin{array}{c}\text { Global } \\
\text { efficiency [-] }\end{array}$ & 0.51 & 0.50 & 0.55 & 0.58 \\
\hline $\begin{array}{c}\text { Indicated } \\
\text { Efficiency [-] }\end{array}$ & 0.81 & 0.79 & 0.79 & 0.85 \\
\hline $\begin{array}{c}\text { Mechanical } \\
\text { Efficiency [-] }\end{array}$ & 0.65 & 0.67 & 0.72 & 0.72 \\
\hline $\begin{array}{c}\text { Volumetric } \\
\text { efficiency [-] }\end{array}$ & 0.97 & 0.95 & 0.95 \\
\hline
\end{tabular}

The results show as the best efficiency is achieved with LSDAP (Case D), hence adopting the lower revolution speed LS solution with the dual axial port configuration DAP (Figure 2(b)) In this case in fact, the global efficiency in correspondence of the design point is equal to 0.58 thanks to a high indicated efficiency $(0.85)$ and an acceptable mechanical performance (0.72). Concerning the other solution, case B (HSDAP) presents the lowest global efficiency $(0.50)$ mainly due to friction losses which leads to a mechanical efficiency of 0.67 as the indicated efficiency value $(0.79)$ can be retained satisfactory for this application. The adoption of radial port to the high speed solution (case A- HSRP) provides a slight improvement of the efficiency (0.51) boosting the indicated efficiency up to (0.81). Nevertheless, in this case the reduction of the indicated power is not accompanied by a reduction of the mechanical losses and consequently the global impact on pump efficiency increases, providing a reduction of the mechanical efficiency (0.65). The case C (LSRP) presents the maximum mechanical efficiency $(0.72)$ thanks to the lower revolution speed. Nevertheless, the indicated efficiency is smaller with respect the LSDAP port case $(0.79)$ thus providing that the global efficiency is equal to 0.55 . Consequently, these results show as the choice to reduce the revolution speed is an interesting solution to contain the mechanical losses. Furthermore, the adoption of dual axial port ensures to provide an effective intake and exhaust ports. Radial ports allow also to achieve a satisfactory indicated efficiency (0.79-0.81). Therefore, low speed dual axial port LSDAP pump was selected (case D) among the considered configuration since it ensures the achievement of the best performances. 
Figure 3. SVRP characteristic curves of LSDAP in terms of Q- $\Delta p$ (a), $\Delta p-\eta_{\text {glob }}(\mathrm{b}), \Delta \mathrm{p}-\eta_{\text {mech }}$ (c) $\Delta \mathrm{p}$ $\eta_{\text {ind }}(\mathrm{d})$

\begin{tabular}{|rrr|}
\hline $250 \mathrm{rpm}$ & $1150 \mathrm{rpm}$ & \multicolumn{2}{c|}{$2050 \mathrm{rpm}$} \\
$700 \mathrm{rpm}$ & $1600 \mathrm{rpm}$ & -- ICE hydraulic impedance \\
\hline
\end{tabular}

(a)

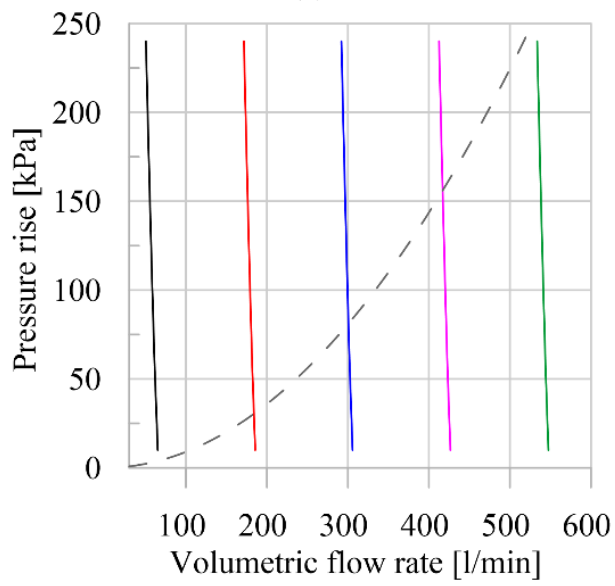

(c)

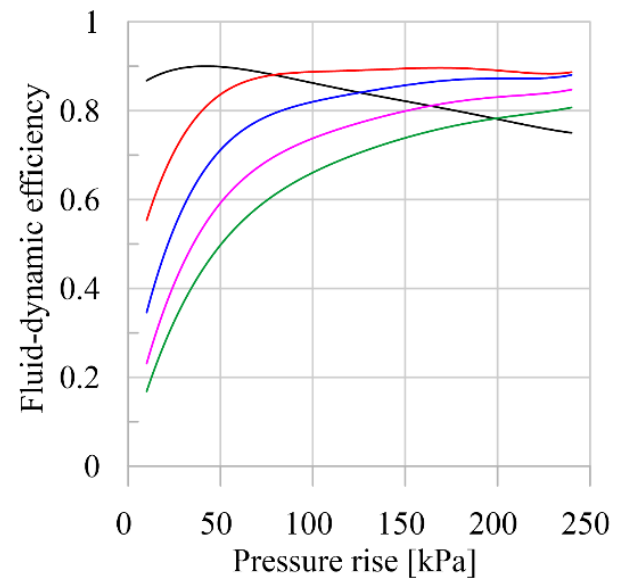

(b)

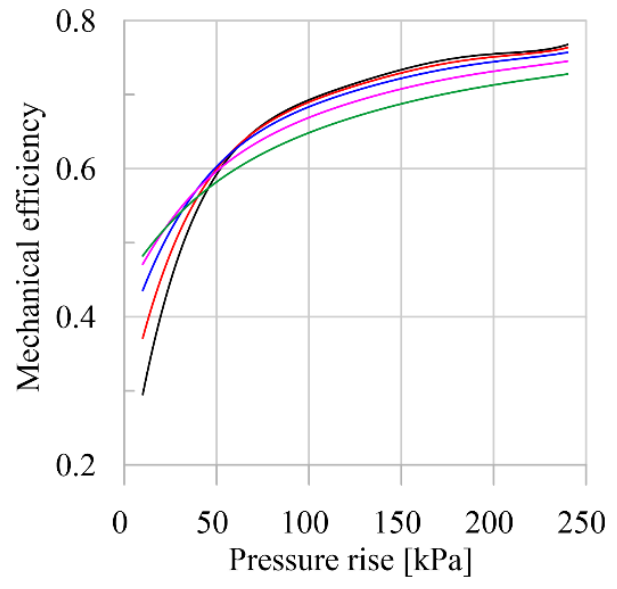

(d)

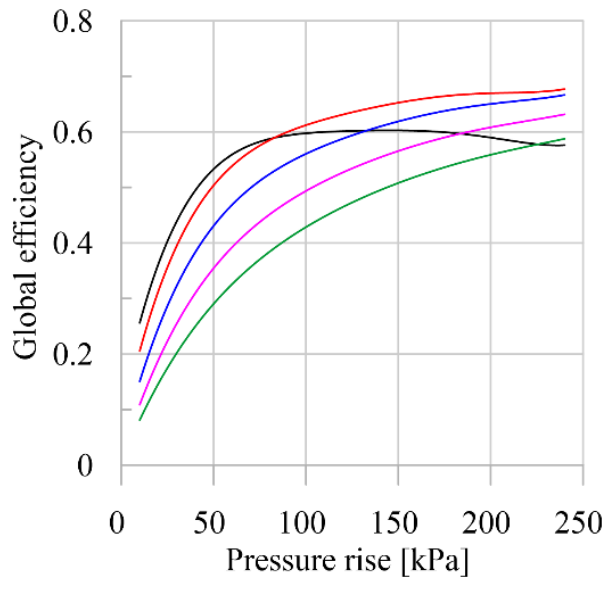

In Figure 3(a) the characteristic curve Q- $\Delta$ p of LSDAP are reported for $250 \mathrm{rpm}, 700 \mathrm{rpm}$, $1150 \mathrm{rpm}, 1600 \mathrm{rpm}$ and $2050 \mathrm{rpm}$. The slopes of the curves (almost straight lines) indicate the high volumetric efficiency of the pump. The figure shows that LSDAP can cover a wide flow rate operating range $(30-5501 / \mathrm{min})$ and pressure rise $(2-250 \mathrm{kPa})$ in a wide range of revolution speed (250-2050 RMP). This speed range reduces the friction losses consequently achieving a high mechanical efficiency (0.6-0.78) for a huge pressure rise interval $(50-250$ $\mathrm{kPa}$ ). The mechanical efficiency (Figure $3(\mathrm{~b})$ ) shows a reduction only for a low pressure rise $(<50 \mathrm{kPa})$. Indeed, in this case the hydraulic power is small while friction losses are mostly related to the revolution speed. Anyway, in this critical region the machine guarantees a mechanical efficiency from 0.5 to 0.3 decreasing $\omega$ from $2050 \mathrm{rpm}$ to $250 \mathrm{rpm}$. Concerning the fluid-dynamic efficiency (Figure 3(c)) given by the product between indicated and 
volumetric ones, it increases when the revolution speed decreases. This is due to the better filling and emptying.

However, also when LSDAP rotates at $1600 \mathrm{rpm}$ the fluid-dynamic efficiency varies between 0.6 to 0.83 for a pressure increase within the $50 \mathrm{kPa}-250 \mathrm{kPa}$ interval. When the pressure rise is lower than 0.5 the indicated efficiency decreases up to 0.2 due to the low hydraulic power. The indicated and mechanical efficiencies drive the trend of the global efficiency which, for all the cases, increases with pressure rise. This is due to the increase of the hydraulic power which decrease the impact of mechanical and fluid-dynamic losses. As it was expected, a positive benefit on global efficiency is also played by the reduction of the revolution speed which anyway should not assume too small values. In fact, the maximum global efficiency values (up to 0.64) are achieved for intermediate revolution speeds (in the range of 700-1600 $\mathrm{rpm})$. This means that a further improvement of the efficiency can be provided increasing the pump displaced volume which allows a reduction of the revolution speed. In any case, the pump achieves high efficiency (0.4-0.65) for a wide operating range. Thherefore, even though the machine works far from the design point, slight global efficiency penalties are observed.

The key aspect that makes this approach suitable is that the displacement increase can be obtained with a small increase of the radial dimension of the machine. Hence, this design choice does not provide a sensible increase of pump dimensions.

\section{Conclusions}

In the present work a design of a Sliding Rotary Vane Pump for the cooling of the 131 heavy duty internal combustion engine (FPT Industrial Cursor 13-NG) has been done. This made use of a comprehensive model of the Sliding Rotary Vane Pump used as model-based design tool.

The first step regarded a preliminary design analytically performed in which two possible configurations are considered:

- $\quad$ High speed SVRP pump (2250 rpm) with a lower displaced volume 0.219 l;

- $\quad$ Low speed SVRP pump (1800 rpm) with higher displaced volume 0.273 1;

For both revolution speeds, radial and dual axial port configurations are considered.

The results show that the best efficiency is reached considering the lower design speed with a dual axial port configuration (LSDAP). Indeed, the low revolution speed produces an improvement of mechanical efficiency $(0.72)$ whereas the dual axial port configuration guarantees a proper intake and exhaust phase as demonstrated by the high indicated efficiency (0.88). The optimization of mechanical and indicated efficiency has as whole effect the optimization of global efficiency which for Low Speed Dual Axial Port (LSDAP) Pump solution assumes a value equal to 0.6 . The other solutions present respectively global efficiency equal to:

- 0.44 for High Speed Radial Port Pump;

- 0.54 for High Speed Dual Axial Port Pump;

- 0.52 for Low Speed Radial Port Pump;

For this reason, LSDAP is chosen, and its performance was evaluated also in a wide operating range. The results demonstrate: 
- The machine can cover a wide operating range (30-550 $\mathrm{l} / \mathrm{min})$ with a contained revolution speed variation;

- $\quad$ The global efficiency varies between 0.4 and 0.65 for a huge pressure rise (0.5-2.5);

- For lower pressure rise the global efficiency decreases but these penalties can be overcome further reducing the revolution speed.

Therefore, it can be concluded as the design of sliding rotary vane pump should be oriented to a reduction of revolution speed obtained by the enhancement of displaced volume. The crucial aspect that makes this strategy feasible is that a slight increase of stator diameter $(+$ $3 \%)$ produces a sensible growth of displaced volume $(+25 \%)$.

\section{Nomenclature}

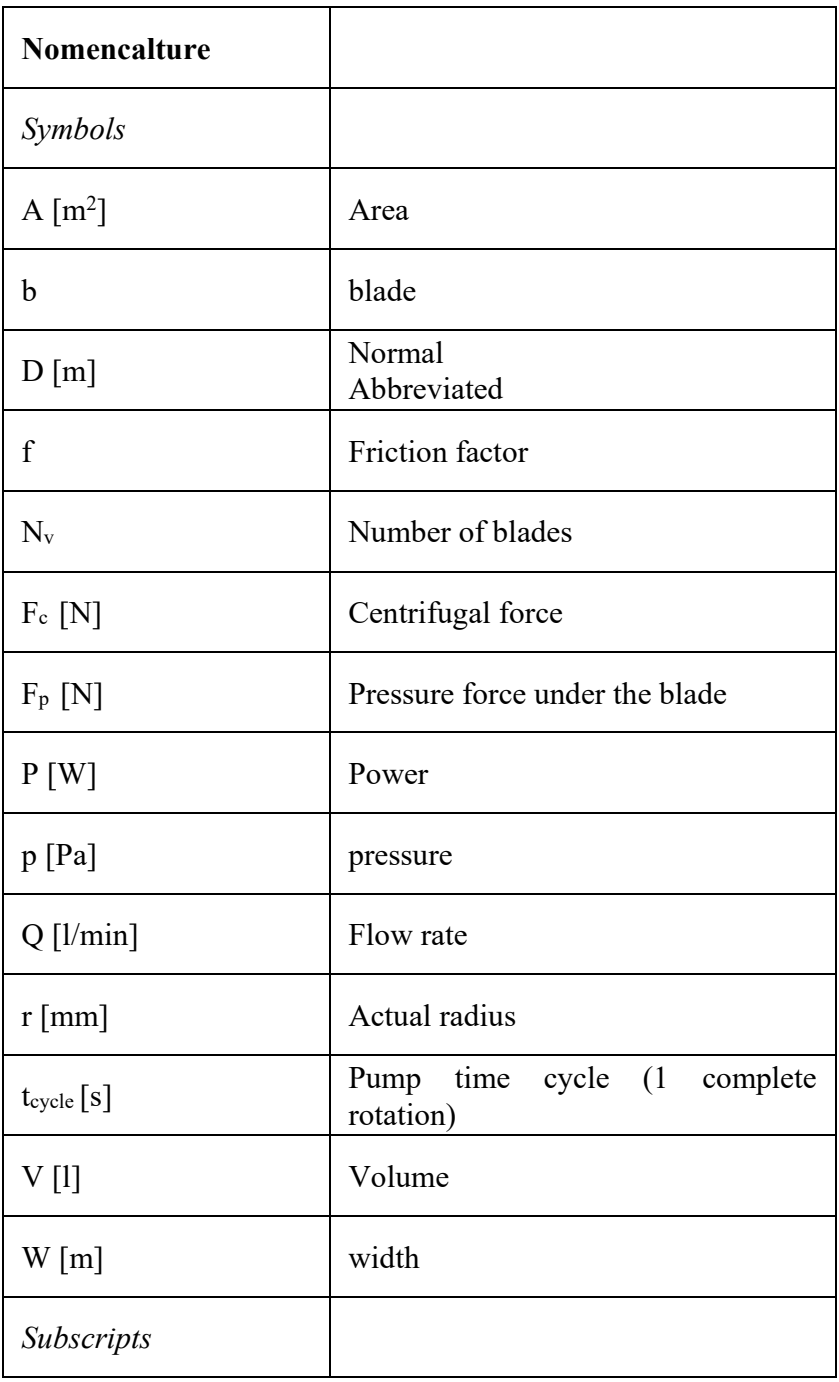




\begin{tabular}{|c|c|}
\hline $\mathrm{amb}$ & ambient pressure \\
\hline disp & displaced \\
\hline hyd & Hydraulic \\
\hline glob & Global \\
\hline ind & indicated \\
\hline mech & mechanical \\
\hline 1 & length \\
\hline losses & losses due to friction \\
\hline $\mathrm{rt}$ & rotor \\
\hline st & stator \\
\hline $\mathrm{t}$ & thick \\
\hline th & theoretical \\
\hline $\mathrm{ub}$ & Under the blades \\
\hline vol & volumetric \\
\hline \multicolumn{2}{|c|}{ Greek Letters } \\
\hline$\Delta \mathrm{p}[\mathrm{Pa}]$ & Pressure rise \\
\hline$\eta$ & efficiency \\
\hline$\omega[\mathrm{rpm}]$ & Revolution speed \\
\hline \multicolumn{2}{|c|}{ Acronyms } \\
\hline HSDAP & High Speed Dual Axial Ports \\
\hline HSRP & High Speed Dual Axial Ports \\
\hline LSDAP & Low Speed Radial Port \\
\hline LSDAP & Low Speed Dual Axial Port \\
\hline ICE & Internal Combustion Engine \\
\hline SVRP & Sliding Rotary Vane Pump \\
\hline
\end{tabular}




\section{Acknowledgments}

The current research work has been developed in the framework of the EU project "LONGRUN - Development of efficient and environmental friendly LONG distance powertrain for heavy duty trucks and coaches". The authors are gratefully to $\mathrm{CNH}$ Industrial S.p.A and to O.M.P. Officine Mazzocco Pagnoni S.r.L. for the precious support during the activity.

\section{References}

[1] EEA, "Carbon dioxide emissions from Europe' s heavy-duty vehicles,” 2018.

[2] EU, Regulation (EU) 2019/1242 of the European Parliament and of the Council of 20 June 2019 Setting CO2 emission performance standards for new heavy-duty vehicles and amending Regulations (EC) No 595/2009 and (EU) 2018/956 of the European Parliament, vol. L 198, no. April. 2019, pp. 202-240.

[3] W. Li, L. Ji, L. Ma, Y. Yang, L. Zhou, and R. K. Agarwal, "Numerical and experimental study of variable speed automobile engine cooling water pump," Sci. Prog., vol. 103, no. 2, pp. 1-27, 2020.

[4] W. Li, E. Li, W. Shi, W. Li, and X. Xu, "Numerical simulation of cavitation performance in engine cooling water pump based on a corrected cavitation model," Processes, vol. 8, no. 3, 2020.

[5] W. Li, X. Zhao, W. Li, W. Shi, L. Ji, and L. Zhou, "Numerical Prediction and Performance Experiment in an Engine Cooling Water Pump with Different Blade Outlet Widths," Math. Probl. Eng., vol. 2017, 2017.

[6] K. Arunachalam and P. M. Jawahar, "Conversion of Mechanical Water Pump to Electric Water Pump for a CI Engine,” vol. 8, no. 12, pp. 2049-2052, 2014.

[7] H. Cho, D. Jung, Z. S. Filipi, D. N. Assanis, J. Vanderslice, and W. Bryzik, "Application of controllable electric coolant pump for fuel economy and cooling performance improvement," J. Eng. Gas Turbines Power, vol. 129, no. 1, pp. 239244, 2007.

[8] X. Wang, X. Liang, Z. Hao, and R. Chen, "Comparison of electrical and mechanical water pump performance in internal combustion engine," Int. J. Veh. Syst. Model. Test., vol. 10, no. 3, pp. 205-223, 2015.

[9] S. Loganathan, S. Govindarajan, J. Suresh Kumar, K. Vijayakumar, and K. Srinivasan, "Design and development of vane type variable flow oil pump for automotive application," SAE Tech. Pap., 2011.

[10] M. Rundo, "Piloted displacement controls for ICE lubricating vane pumps," SAE Int. J. Fuels Lubr., vol. 2, no. 2, pp. 176-184, 2010.

[11] B. Geist, W. Resh, and K. Aluru, "Calibrating an adaptive pivoting vane pump to deliver a stepped pressure profile," SAE Tech. Pap., vol. 2, 2013.

[12] P. E. Sullivan and M. Sehmby, "Internal force analysis of a variable displacement 
vane pump," SAE Tech. Pap., 2012.

[13] N. Novi, R. Squarcini, and F. Frendo, "Dynamic and kinematic evaluation of automotive variable displacement vane pumps for reliability characterization," $S A E$ Tech. Pap., 2009.

[14] Y. Inaguma and N. Yoshida, "Variation in driving torque and vane friction torque in a balanced vane pump," SAE Tech. Pap., vol. 1, 2014.

[15] Y. Inaguma and N. Yoshida, "Small High-Efficiency Vane Pump Based on Vane Pump Theory," SAE Int. J. Passeng. Cars - Mech. Syst., vol. 8, no. 2, pp. 614-623, 2015.

[16] J. Harrison, R. Aihara, M. Eshraghi, and I. Dmitrieva, "Modeling engine oil variable displacement vane pumps in $1 \mathrm{~d}$ to predict performance, pulsations, and friction," SAE Tech. Pap., vol. 1, 2014.

[17] F. Fatigati, M. Di Bartolomeo, G. Lo Biundo, F. Pallante, and R. Cipollone, "Theoretical and experimental control strategies assessment of a Sliding Vane Oil Pump,” E3S Web Conf., vol. 197, p. 06022, 2020.

[18] S. Jayanthamani, R. Sivanantham, and M. Ibrahim, "Mathematical Modelling and Analysis of Vane Type Variable Displacement Oil Pump," SAE Tech. Pap., 2019.

[19] R. Cipollone and D. Di Battista, "Sliding vane rotary pump in engine cooling system for automotive sector," Appl. Therm. Eng., vol. 76, pp. 157-166, 2015.

[20] R. Cipollone, G. Bianchi, D. Di Battista, and F. Fatigati, "Fuel economy benefits of a new engine cooling pump based on sliding vane technology with variable eccentricity," Energy Procedia, vol. 82, pp. 265-272, 2015.

[21] F. Fatigati, D. Di Battista, and R. Cipollone, "Design improvement of volumetric pump for engine cooling in the transportation sector," Energy, vol. 231, p. 120936, 2021.

[22] F. Fatigati, M. Di Bartolomeo, D.Di Battista, R. Cipollone, Model based control of the inlet pressure of a sliding vane rotary expander operating in an ORC-based power unit, Applied Thermal Engineering, vol. 193, p. 117032, 2021 